Case Report

\title{
Prefibrotic Myelofibrosis Presenting with Multiple Cerebral Embolic Infarcts and the Rare MPL W515S Mutation
}

\author{
Stephen E. Langabeer ${ }^{(D},{ }^{1}$ Lisa Lee Tokar, ${ }^{1}$ Laura Kearney, ${ }^{1}$ Cathal O'Brien, ${ }^{1}$ \\ Kowshika Thavarajah, ${ }^{2}$ Aisling Barrett, ${ }^{3}$ John McManus, ${ }^{2}$ and Hilary O'Leary ${ }^{3}$ \\ ${ }^{1}$ Cancer Molecular Diagnostics, St. James's Hospital, Dublin D08 W9RT, Ireland \\ ${ }^{2}$ Department of Medicine for the Elderly, University Hospital Limerick, Limerick V94 F858, Ireland \\ ${ }^{3}$ Department of Haematology, University Hospital Limerick, Limerick V94 F858, Ireland \\ Correspondence should be addressed to Stephen E. Langabeer; slangabeer@stjames.ie
}

Received 22 January 2020; Revised 24 May 2020; Accepted 4 June 2020; Published 20 June 2020

Academic Editor: Pier Paolo Piccaluga

Copyright ( 2020 Stephen E. Langabeer et al. This is an open access article distributed under the Creative Commons Attribution License, which permits unrestricted use, distribution, and reproduction in any medium, provided the original work is properly cited.

\begin{abstract}
Acquired, activating mutations of MPL W515 are recognised driver mutations of the myeloproliferative neoplasms (MPN), namely, essential thrombocythemia and primary myelofibrosis. The most common mutation at this codon is W515L with several other mutations also described at a lower frequency. Of these less common mutations, MPL W515S has only been reported sporadically with limited information on clinicopathological associations. We describe the case of an elderly man with persistent thrombocytosis presenting with an ischemic cerebral event. Bone marrow biopsy showed evidence of prefibrotic myelofibrosis with targeted sequencing demonstrating the presence of the rare MPL W515S mutation. Thrombolytic and cytoreductive therapies resulted in a favorable outcome and follow-up. This case provides additional, necessary, and phenotypic data for the rare MPNassociated MPL W515S mutation.
\end{abstract}

\section{Introduction}

The most commonly acquired driver mutations of the Philadelphia chromosome-negative myeloproliferative neoplasms (MPN) of essential thrombocythemia (ET) and primary myelofibrosis (PMF) are those of JAK2 V617F, CALR exon 9, and MPL exon 10. Identification of these mutations constitutes a major diagnostic criterion for disease diagnosis and classification [1]. MPL encodes the receptor for thrombopoietin with the first MPN-associated, MPL exon 10 mutation described as W515L swiftly followed by reports of several other mutations occurring at this particular tryptophan (Trp) codon in patients with both ET and PMF [2-4]. However, the W515 codon appears to be a hotspot for acquired mutations, and several other somatic and inherited variants of $M P L$ (other than in exon 10) have also been annotated in familial and sporadic MPN [5-9].

Morphological comparison of $M P L$-mutated ET with JAK2 V617F- and CALR-mutated ET has shown that
MPL-mutated ET patients are older than those with other genotypes and have higher peripheral blood platelet counts and bone marrow megakaryocytes than JAK2 V617F-mutated ET with histological features of prefibrotic myelofibrosis being rare [10]. Review of bone marrow histology of MPL-mutated PMF shows decreased vascularity and increased osteosclerosis [11]. Clinically, MPL-mutated MPN patients tend to have lower risk of thrombotic events than their JAK2 V617F counterparts [12-14].

The W515S mutation was first described in in vitro as a spontaneously occurring point mutation in the intracellular domain of the protein after transfection of $c$ - $m p l$ into $\mathrm{Ba} / \mathrm{F} 3$ cells that resulted in thrombopoietin-independent, constitutive activation of the receptor [15]. Isolated reports of $M P L$ W515S in MPN patients exist in the literature, usually within a cohort, yet limited clinical and pathological associations regarding this specific mutation exist [16-19]. Of these four cases, two patients had annotated clinical histories and in whom no thrombotic episodes were observed. 


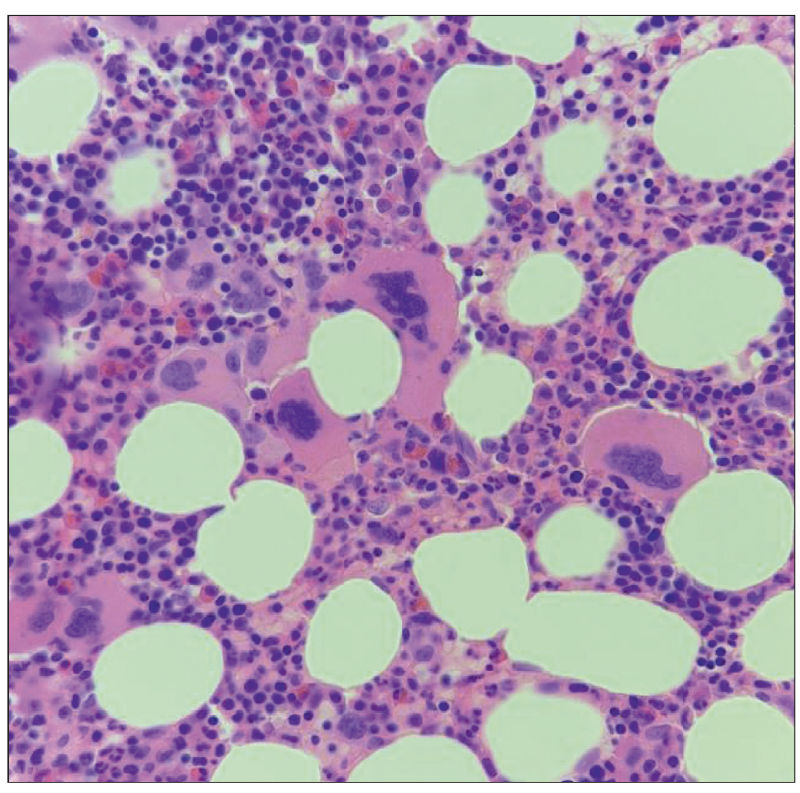

(a)

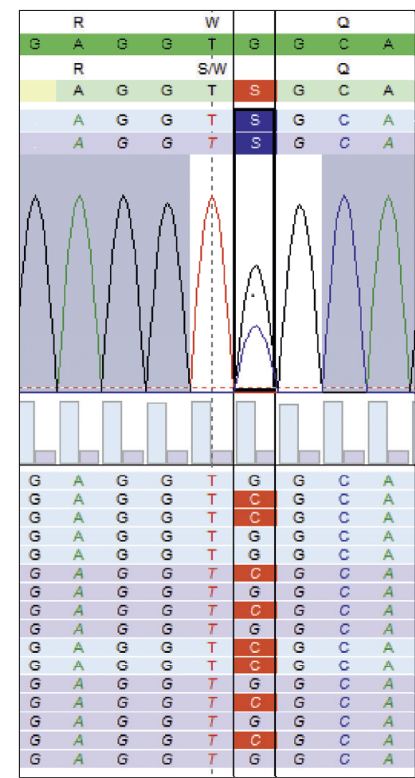

(b)

FIgURE 1: (a) Bone marrow biopsy demonstrating megakaryocyte clustering with nuclear hypolobation and bulbous forms; (b) nextgeneration sequence analysis (Sequence Pilot, JSI Medical Systems, Ettenheim, Germany) identifying MPL p.W515S (c.1544G > C).

\section{Case Report}

A 71-year-old man with a persistent high platelet count for four years (range $483-797 \times 10^{9} / \mathrm{L}$ ) presented with dense, right-sided weakness, aphasia, and a decreased level of consciousness. He was known to have hypercholesterolemia for which he was on atorvastatin. He was an ex-smoker for forty years and otherwise did not have any other vascular risk factors. Apart from thrombocytosis, blood count parameters including hemoglobin, hematocrit, white cell count, and white cell differential had always been normal. Initial computerized tomography (CT) scan showed an old, left occipital lobe infarction. CT angiogram did not show any large vessel occlusion. Magnetic resonance imaging scan confirmed multiple, left-sided, and embolic infarcts. Of note, a 72-hour Holter monitor excluded paroxysmal atrial fibrillation as a cause of cerebral ischemia. Standard stroke workup did not identify any other sources of cerebral embolic infarction. He was thrombolysed and subsequently commenced on low-dose aspirin $75 \mathrm{mg}$ daily. He made an excellent recovery, the only residual deficit being that of slightly altered speech.

Following his discharge from the hospital, he was referred to the hematology service for investigation of the persistent thrombocytosis. Bone marrow aspirate was aparticulate. Bone marrow biopsy was hypercellular with increased numbers of megakaryocytes showing paratrabecular clustering and nuclear hypolobation as well as bulbous forms (Figure 1(a)); granulopoiesis and erythropoiesis looked normal with World Health Organization grade 1 reticulin fibrosis seen that are all features in keeping with a diagnosis of prefibrotic myelofibrosis. An International Prognostic Scoring System score of 1 predicted his median overall survival for more than 10 years [20].
Karyotypic analysis failed on the bone marrow aspirate; however, a next-generation sequencing approach identified p.(Trp515Ser), c.1544G > C mutation (NM_005373.2), usually referred to as $M P L \mathrm{~W} 515 \mathrm{~S}$, at an allele frequency of $35 \%$ in the patient's peripheral blood (Figure 1(b)). No CALR exon 9 or JAK2 exon 12 or 14 mutations were identified.

In order to reduce the risk of further thrombotic events in the context of confirmed MPL W515S MPN, hydroxyurea $500 \mathrm{mg}$ daily was introduced, resulting in the platelet count normalising within one month of starting this treatment and remaining to date within the normal range. Currently, the patient remains well and is fully capable of carrying out all daily activities. He has a persisting, slight speech impediment and remains on aspirin and hydroxyurea more than twelve months following the ischaemic cerebral event and has not had any bleeding sequelae.

\section{Discussion}

The Trp515 amino acid is located at the transmembranecytosolic junction of the MPL receptor controlling dimerization and activation [21]. This codon is recurrently mutated in MPN with the mutations W515L and W515K detected most frequently but with W5151R, W515A, and W515G also described [16, 22], whereas MPL W515S has only been sporadically reported in ET and PMF patients [16-19]. In a recent study of 17 active mutants resulting from substitutions at Trp515, W515S resulted in activation of the TpoR/JAK2/STAT5 transcriptional cascade in the absence of the ligand in a similar manner to that of other mutants described in MPN patients thus implicating its oncogenic potential [23].

As MPN are relatively rare, it is probable they represent an underdiagnosed cause of cerebral thrombosis. High 
clinical awareness is warranted to identify an underlying MPN in order that a prompt treatment is initiated (e.g., antiplatelet, cytoreductive, and phlebotomy) [24, 25]. While some studies have suggested routine screening for JAK2 $\mathrm{V} 617 \mathrm{~F}$ in patients presenting with stroke and/or cerebral venous thrombosis (CVT) [26], given the lower risk of thrombosis in CALR- and MPL-mutated MPN patients and the near absence of these mutations in CVT cohorts [27, 28], wider evaluation of mutation status might be reserved for those patients with sustained abnormal blood count findings.

Detection of MPN-associated mutations is achieved by a number of technological approaches including Sanger sequencing, allele-specific PCR, melt curve analysis, capillary electrophoresis, pyrosequencing, digital PCR, and nextgeneration sequencing [29]. Given the possibility of rare mutations such as W515S, a diagnostic screening approach covering at least $M P L$ exon 10 such as next-generation sequencing is preferable to those techniques that target individually the most prevalent mutations of $M P L$ W515L and W515K.

Identification and reporting of such rare $M P L$ mutations in conjunction with the clinical aspects in MPN patients allow for correct diagnosis, assessment of any genotypephenotype relationship, and may provide information on any future therapeutic approaches in similar patients.

\section{Data Availability}

No data were used to support this study.

\section{Conflicts of Interest}

The authors declare that there are no conflicts of interest regarding the publication of this paper.

\section{References}

[1] D. A. Arber, A. Orazi, R. Hasserjian et al., "The 2016 revision to the world health organization classification of myeloid neoplasms and acute leukemia," Blood, vol. 127, no. 20, pp. 2391-2405, 2016.

[2] Y. Pikman, B. H. Lee, T. Mercher et al., "MPL W515L is a novel somatic activating mutation in myelofibrosis with myeloid metaplasia," PLoS Medicine, vol. 3, no. 7, p. e270, 2006.

[3] A. D. Pardanani, R. L. Levine, T. Lasho et al., "MPL515 mutations in myeloproliferative and other myeloid disorders: a study of 1182 patients," Blood, vol. 108, no. 10, pp. 3472-3476, 2006.

[4] R. Chaligné, C. Tonetti, R. Besancenot et al., "New mutations of MPL in primitive myelofibrosis: only the MPL W515 mutations promote a G1/S-phase transition," Leukemia, vol. 22, no. 8, pp. 1557-1566, 2008.

[5] J. D. Milosevic Feenstra, H. Nivarthi, H. Gisslinger et al., "Whole-exome sequencing identifies novel MPL and JAK2 mutations in triple-negative myeloproliferative neoplasms," Blood, vol. 127, no. 3, pp. 325-332, 2016.

[6] X. Cabagnols, F. Favale, F. Pasquier et al., "Presence of atypical thrombopoietin receptor (MPL) mutations in triple-negative essential thrombocythemia patients," Blood, vol. 127, no. 3, pp. 333-342, 2016.

[7] A. Angona, C. Fernández-Rodríguez, A. Alvarez-Larrán et al., "Molecular characterisation of triple negative essential thrombocythaemia patients by platelet analysis and targeted sequencing," Blood Cancer Journal, vol. 6, no. 8, p. e463, 2016.

[8] Y. C. Chang, H. C. Lin, Y. H. Chiang et al., "Targeted nextgeneration sequencing identified novel mutations in triplenegative myeloproliferative neoplasms," Medical Oncology, vol. 34, no. 5, 2017.

[9] S. Schulze, R. Stengel, N. Jaekel et al., "Concomitant and noncanonical JAK2 and MPL mutations in JAK2 V617F- and MPLW 515 L-positive myelofibrosis," Genes, Chromosomes and Cancer, vol. 58, no. 11, pp. 747-755, 2019.

[10] A. Alvarez-Larran, D. Martínez, L. Arenillas et al., "Essential thrombocythaemia with mutation in MPL: clinicopathological correlation and comparison with JAK2V617F-mutated and CALR-mutated genotypes," Journal of Clinical Pathology, vol. 71, no. 11, pp. 975-980, 2018.

[11] W. J. Wong, R. P. Hasserjian, G. S. Pinkus et al., "JAK2, CALR, MPL and ASXL1 mutational status correlates with distinct histological features in Philadelphia chromosome-negative myeloproliferative neoplasms," Haematologica, vol. 103, no. 2, pp. e63-e68, 2018.

[12] A. M. Vannucchi, E. Antonioli, P. Guglielmelli et al., "Characteristics and clinical correlates of MPL 515W $>\mathrm{L} / \mathrm{K}$ mutation in essential thrombocythemia," Blood, vol. 112, no. 3, pp. 844-847, 2008.

[13] N. Gangat, E. A. Wassie, T. L. Lasho et al., "Mutations and thrombosis in essential thrombocythemia: prognostic interaction with age and thrombosis history," European Journal of Haematology, vol. 94, no. 1, pp. 31-36, 2015.

[14] M. C. Finazzi, A. Carobbio, F. Cervantes et al., "CALR mutation, MPL mutation and triple negativity identify patients with the lowest vascular risk in primary myelofibrosis," Leukemia, vol. 29, no. 5, pp. 1209-1210, 2015.

[15] M. Abe, K. Suzuki, O. Inagaki, S. Sassa, and H. Shikama, “A novel MPL point mutation resulting in thrombopoietin-independent activation," Leukemia, vol. 16, no. 8, pp. 15001506, 2002.

[16] W. Ma, X. Zhang, X. Wang et al., "MPL mutation profile in JAK2 mutation-negative patients with myeloproliferative disorders," Diagnostic Molecular Pathology, vol. 20, no. 1, pp. 34-39, 2011.

[17] J. Tao, X. Zghang, J. Lancet et al., "Concurrence of B-lymphoblastic leukemia and myeloproliferative neoplasm with copy neutral loss of heterozygosity at chromosome 1p harbouring a MPL W515S mutation," Cancer Genetics, vol. 2017, no. 10-12, pp. 489-494, 2014.

[18] A. Tefferi, T. L. Lasho, K. H. Begna et al., "A pilot study of the telomerase inhibitor imetelstat for myelofibrosis," New England Journal of Medicine, vol. 373, no. 10, pp. 908-919, 2015.

[19] A. Beucher, M. Dib, C. Orvain et al., "Next generation sequencing redefines a triple negative essential thrombocythaemia as double-positive with rare mutations on JAK 2 V617 and MPL W515 hotspots," British Journal of Haematology, vol. 186, no. 5, pp. 785-788, 2019.

[20] P. Guglielmelli, A. Pacilli, G. Rotunno et al., "Presentation and outcome of patients with 2016 WHO diagnosis of prefibrotic and overt primary myelofibrosis," Blood, vol. 129, no. 24, pp. 3227-3236, 2017.

[21] J.-P. Defour, M. Itaya, V. Gryshkova et al., "Tryptophan at the transmembrane-cytosolic junction modulates thrombopoietin receptor dimerization and activation," Proceedings of the 
National Academy of Sciences, vol. 110, no. 7, pp. 2540-2545, 2013.

[22] J. Z. Gong, J. R. Cook, T. C. Greiner et al., "Laboratory practice guidelines for detecting and reporting JAK2 and MPL mutations in myeloproliferative neoplasms," The Journal of Molecular Diagnostics, vol. 15, no. 6, pp. 733-744, 2013.

[23] J.-P. Defour, I. Chachoua, C. Pecquet, and S. N. Constantinescu, "Oncogenic activation of MPL/ thrombopoietin receptor by 17 mutations at W515: implications for myeloproliferative neoplasms," Leukemia, vol. 30, no. 5, pp. 1214-1216, 2016.

[24] A. Artoni, P. Bucciarelli, and I. Martinelli, "Cerebral thrombosis and myeloproliferative neoplasms," Current Neurology and Neuroscience Reports, vol. 14, no. 11, 2014.

[25] M. I. Stefanou, H. Richter, F. Härtig et al., "Recurrent ischaemic cerebrovascular events as presenting manifestations of myeloproliferative neoplasms," European Journal of Neurology, vol. 26, no. 6, pp. 903-e64, 2019.

[26] M. Lamy, P. Palazzo, P. Agius et al., "Should we screen for janus kinase 2 V617F mutation in cerebral venous thrombosis?" Cerebrovascular Diseases, vol. 44, no. 3-4, pp. 97-104, 2017.

[27] I. Martinelli, V. De Stefano, A. Carobbio et al., "Cerebral vein thrombosis in patients with philadelphia-negative myeloproliferative neoplasms an european leukemia net study," American Journal of Hematology, vol. 89, no. 11, pp. E200E205, 2014.

[28] E. Verger, I. Crassard, B. Cassinat, and S. Bellucci, "Low incidence of CALR gene mutations in patients with cerebral venous thrombosis without overt chronic myeloproliferative neoplasm," Thrombosis Research, vol. 136, no. 4, pp. 839-840, 2015.

[29] S. E. Langabeer, H. Andrikovics, J. Asp et al., "Molecular diagnostics of myeloproliferative neoplasms," European Journal of Haematology, vol. 95, no. 4, pp. 270-279, 2015. 